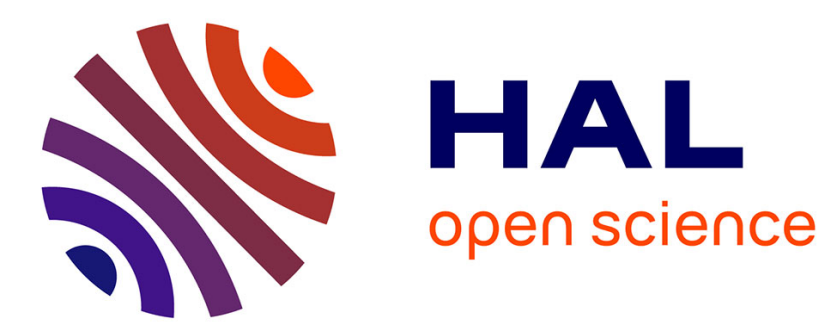

\title{
Apparences et imaginations chez Nicole Oresme : Question III.1 sur la "Physique" et question sur l'apparence d'une chose \\ Jean Celeyrette
}

\section{- To cite this version:}

Jean Celeyrette. Apparences et imaginations chez Nicole Oresme: Question III.1 sur la "Physique" et question sur l'apparence d'une chose. Revue d'Histoire des Sciences, 2007, 60-1 (janvier-juin), p. 83-100. halshs-00181586

\section{HAL Id: halshs-00181586 \\ https://shs.hal.science/halshs-00181586}

Submitted on 2 Jul 2015

HAL is a multi-disciplinary open access archive for the deposit and dissemination of scientific research documents, whether they are published or not. The documents may come from teaching and research institutions in France or abroad, or from public or private research centers.
L'archive ouverte pluridisciplinaire HAL, est destinée au dépôt et à la diffusion de documents scientifiques de niveau recherche, publiés ou non, émanant des établissements d'enseignement et de recherche français ou étrangers, des laboratoires publics ou privés. 
Revue

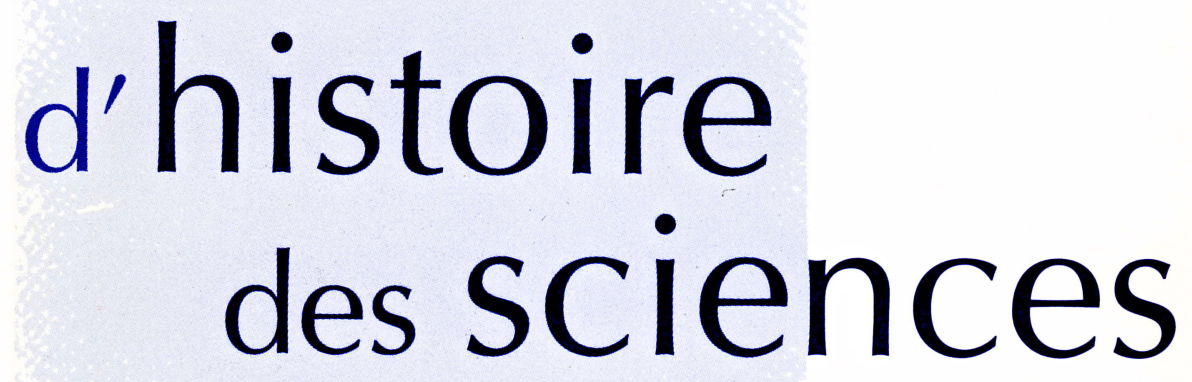

Tome 60-1 | janvier-juin 2007

\section{SCIENCES, TEXTES ET CONTEXTES}

En hommage à Gérard Simon 


\title{
Apparences et imaginations chez Nicole Oresme : Question III.1 sur la Physique et question sur l'apparence d'une chose *
}

Jean CELEYRETTE **

\begin{abstract}
Résumé : Pour Nicole Oresme, toute connaissance provient de la perception sensible. L'analyse comparée de deux de ses questions, la question III.1 sur la Physique et la question "De apparentia rei " montre, sur les exemples du mouvement et de la grandeur, que la perception visuelle ne nous fournit que très peu de certitudes. Dans ces conditions, le recours à des perceptions imaginaires, loin d'être un jeu mathématique gratuit, est un moyen d'élargir le champ du connaissable.
\end{abstract}

Mots-clés : vision ; certitude ; mouvement ; grandeur.

\begin{abstract}
Summary : For Nicole Oresme all knowledge comes from sense perc eptions. A comparative analysis of two of his questions, question III.1 on the Physics and question "de apparentia rei ", shows that visual perception gives us very few certainties. So to resort to imaginary perceptions, far from being a gratuitous mathematical game, is a means for enlarging the scope of the knowable.
\end{abstract}

Keywords : vision ; certainty ; motion ; magnitude.

Les apparences nous sont fournies par nos sens, externes et internes, et c'est à partir d'elles que nous connaissons les choses; leur étude renvoie donc à celle de la connaissance en général. Par ailleurs le sens dont il est question étant le plus souvent celui de la vue, l'étude des apparences est intimement liée à celle des erreurs visuelles, c'est-à-dire de tout ce qui fait que notre vue nous trompe sur la réalité (couleur, forme, emplacement, grandeur, etc.).

Ce sont ces deux aspects que Nicole Oresme développe dans les textes dont nous allons parler.

\footnotetext{
* Je remercie Max Lejbowicz pour ses observatıons, toutes pertınentes, quı m'ont permıs d'amélıorer cet artıcle.

** Jean Celeyrette, UMR "Savoirs, textes, langage " (CNRS-Université de Lille III), Maıson de la recherche, Unıversité de Lılle 3, BP 60149, 59653 Villeneuve d'Asc q Cedex
} 
Les erreurs visuelles constituent un chapitre important des principaux ouvrages d'optique ${ }^{1}$ antique ou médiévale - l'Optique de Ptolémée, le De aspectibus (Sur les apparences) d'Alhazen, les Perspectives de Vitellion et des autres perspectivistes médiévaux -, chapitre qui comprend l'explication technique de leur production, que la théorie adoptée soit le rayon visuel, le rayon lumineux ou un processus mixte ${ }^{2}$.

Les débats sur la connaissance en général ont donné lieu à de nombreuses études, mais l'accent a surtout été mis sur la période précédant les années 1340. Dans son ouvrage de référence, Katherine Tachau ne traite de la situation parisienne au milieu du siècle qu'à travers Grégoire de Rimini, qui a commenté les Sentences en 1343-1344, et Nicolas d'Autrécourt, dont I'Exigit ordo est daté des années 1330. Nicole Oresme ne fait l'objet que d'une brève remarque sur le fait qu'il a probablement subi l'influence des maîtres anglais ${ }^{3}$. En fait, la question de la connaissance a une grande importance dans la philosophie naturelle du maître normand : plusieurs de ses questions sur la Physique ${ }^{4}$ en traitent de façon approfondie, notamment la question III.1 dont nous parlerons et surtout la question I.2 «Est-ce que tout objet causé connaissable est connu par ses causes ${ }^{5}$ ? " II en est de même pour plusieurs de ses questions sur le De anima, notamment la question I.4 ( Est-ce que

1 - Dans l'Antıquité, "un ouvrage d'optıque est une descrıptıon quantıfiée des règles auxquelles obeit le regard" C'est donc un ouvrage sur la vision; l'ımage n'a pas d'existence en dehors de l'observateur : ". . ce qu'un Ancien voit dans un mirorr, c'est la chose même ; maıs là oú elle n'est pas, et comme elle n'est pas " (Cérard Sımon, Le Regard, l'être et l'apparence dans l'optıque de l'Antıquité (Parıs · Seuil, 1988), 196-197.) Au XIV" siècle (hrétien, la question de l'existence de l'ımage est très discutée.

2 - La biblıographie sur le sujet est considérable, et ıl est ımpossible d'en donner icı ne serait-ce qu'une idée (On pourra se reporter à David Lındberg, Roger Bacon and the origins of Perspectiva in the Middle Ages (Oxford · Clarendon Press, 1996), 393-403 À partır de Roger Bacon et de Vitellıon, la science de l'optıque, donc de la visıon, est appelée perspective

3 - Katherine Tachau, Vision and certitude in the age of () ckham - Optic s, epistemology and the foundations of semantics, 1250-1345 (Leiden - New York - Copenhague - Cologne : Brıll, 1988). Voır plus récemment Dallas Denery II, Seeıng and being seen in the later medieval world Optics, theology and religious life (Cambridge : Cambridge Univ. Press, 2005), qui étudie troıs auteurs parisiens, Pierre de Limoges, Pierre Auriol et Nicolas d'Autréc ourt Nicole Oresme n'est pas mentıonné

4 - Ces (Questıones super Physic am d'Cresme, rédıgées au milıeu des années 1340, ne sont conservées que dans le manuscrit Séville, bıblıteca capıtular Colombına, $7630,1 \mathrm{r}^{\circ}$. $79 v^{\circ}$ Les questıons sont classıquement désıgnées par le numéro du livre suıvı du numéro de la question ; aınsı la question III 1 est la premiere question sur le livre III de la Physique d'Aristote

5. "Utrum omne scibıle habens causas sciatur per ıllas?"(Oresme, op cit in $\mathrm{n} 4,1 \mathrm{r}^{\circ} \mathrm{b}$ $\left.v^{\circ} \mathrm{b}\right)$ 
la connaissance des accidents contribue à la connaissance de la substance ? "), et pour la première partie du texte communément appelé De causis mirabilium (Sur les causes des choses extraordinaires) ${ }^{6}$, etc. Par ailleurs il est maintenant bien connu que le thème des illusions visuelles lui a fourni une batterie d'arguments dans des domaines variés. À titre d'exemple, dans son traité sur les configurations des qualités et des mouvements, les apparitions et les songes sont expliqués par l'assimilation de l'âme à un miroir plus ou moins régulier, plus ou moins trouble ${ }^{7}$.

Comme il est impossible de prendre en compte la totalité du corpus oresmien, l'étude qui suit portera sur la question "De apparentia rei » (De I'apparence d'une chose), d'une part parce qu'elle est très peu connue ${ }^{8}$, ensuite parce que le thème de l'apparence constitue l'objet même de l'étude et n'intervient pas seulement de façon incidente ou à titre d'argument. La question posée étant : "Est-ce qu'on voit une chose aussi grande qu'elle est ? ", un rapprochement s'impose avec la deuxième partie intitulée "De quelle façon le mouvement est-il connu? » de la question III.1 sur la Physique ${ }^{9}$. Il pourrait se faire aussi avec la question «Est-ce que les étoiles sont vues là où elles sont?", connue sous le titre "De visione stellarum» (Sur la vision des étoiles). Les problèmes traités sont voisins, et le parallélisme entre les premières séries de conclusions est

6 - Les questıons sur le De anıma ont été éditées dans Peter Marshall, "Nicholas Oresme's Questiones super libros Arıstotelis De anıma A critical edition with introduction and commentary ", thèse de doctorat (Cornell Unıv, 1980) Cette éditıon a été reprıse et complétée dans Benoît Patar, Nicolaı Oresme expositıo et quaestıones in Arıstotelıs De anima (Louvaın-la-Neuve: Éd de l'Instıtut supérıeur de phılosophıe et Louvaın-Parıs: Peeters, 1995). L'éditıon et la traduction anglaıse du De causıs mirabilıum se trouvent dans Bert Hansen, Nicole Oresme and the marvels of nature The De causis mırabilıum (Toronto : Pontifical Institute of mediaeval studies, 1985).

7 - Cf. Marshall Clagett, Nicole Oresme and the geometry of qualitıes and motıons (MadisonMilwaukee-London The Unıv of Wisconsın Press, 1968), part I, chap 33-36, 252-260

8 - Cette questıon a faıt l'objet d'une thèse que je $\mathrm{n}^{\prime}$ aı pas pu consulter: John Watson, "Questiones de apparentıa reı - A hitherto unedited fourteenth-century scientific treatıse ascribed to Nicholas Oresme ", thèse de doctorat (Cambridge, Massachussets, Unıversity of Harvard, 1973). En fait, elle n'a jusqu'à présent guère suscité d'ıntérêt du fait que l'auteur de la thèse a consıdéré son attributıon à (Oresme, mentıonnée dans l'explıcit d'un des tross manuscrits, comme douteuse. Or dans un manuscrit de Venıse, j'aı constaté qu'un maître italıen de la fin du XIV siècle, Marsıle de Saınte-Sophie, cite ce texte et l'attrıbue explıcıtement à Oresme : manuscrit Venıse, bıblıoteca Marciana VI, 72, f. 155 $\mathrm{r}^{\circ} \mathrm{b}-162 \mathrm{v}^{\circ} \mathrm{b}$. En outre la comparaison avec d'autres textes d'Oresme - dont les questıons sur la Physıque - confirme l'attribution

9 - La questıon est divisée en deux partıes. La premıère traıte la questıon du rapport entre la connasssance de la nature et celle du mouvement en posant comme hypothèse que le mouvement est connu, la seconde, "De quelle façon le mouvement est-ıl connu?" (Quomodo motus cognosc Itur), s'interroge sur cette hypothèse. 
frappant. Les trois questions se réfèrent à la Perspective de Vitellion ${ }^{10}$ : les deux premières aux livres III et IV sur la vision directe le livre III traite du processus qui va de I'appréhension immédiate par le sens au jugement par l'âme, et le livre IV des causes des erreurs visuelles -, la troisième question au livre $X$ sur la réflexion et la réfraction. Précisons que l'attribution à Oresme du De visione stellarum n'est pas unanimement acceptée, mais la cohérence avec les deux autres questions, ajoutée aux éléments matériels 11, confirme, me semble-t-il, cette attribution. Toutefois comme cette question a fait l'objet d'une thèse récente ${ }^{12}$, dans laquelle d'ailleurs l'attribution à Oresme n'est pas mise en doute, je n'en parlerai pas davantage, bornant mon étude à la "Questio de apparentia rei » et à la question III.1 sur la Physique.

\section{La question III.1 sur la Physique ${ }^{13}$}

Oresme commence par reprendre de Vitellion (qui le reprend $d^{\prime}$ Alhazen) la distinction entre les sensibles qui sont connus par le sens extérieur de la vue, parmi lesquels seules lumière (lux) ${ }^{14}$ et couleur sont visibles par soi, et les sensibles dont la connaissance

10 - Vitellıon a éc rit son traıté sous une forme d'ınspıratıon euc lıdıenne (axıomes, définıtıons, propositions) et en cela il se distıngue du De aspectibus d'Alhazen et du traité de Roger Bac on (ècrit vers 1268), même sı sur le fond Il est peu orıgınal. Il est écrit entre 1270 et 1274 à la cour clu pape, dans l'entourage de Gullaume de Moerbeke et son succès est très ımportant : dans les textes mèdiévaux traitant de perspectıve, Il est le plus souvent la référence Cf Mark Smith, Witelonis perspectivae liber quintus (Wroclaw-VarsovieCracovie-Cidansk: (Ossolıneum, 1983), "Studıa copernıc ana "XXIII, 13-72.

11 - Il s'agıt pour l'essentıel - 1/ de l'attributıon explıcite à Oresme dans un des troıs manuscrits conservés ; 2 / de l'identité des huit conc lusıons de la seconde partıe avec sa question III 12 sur les Météorologıques.

12 - Danny Burton, "Nicole (Oresme's "( On seeıng the stars" (De visıone stellarum) : A critical edition of Oresme's Treatise on optics and atmospheric refraction, with an introduction, commentary, and Englısh translatıon ", thèse de doctorat (Bloomıngton, Indıana Unıv., 2000)

13 - "Utrum ignorato motu nec esse sit ignorare naturam?" (Est-ce que si l'on ignore le mouvement, néc essarrement on Ignore la nature ?) (Oresme, op. Cit. In n. $4,29 \mathrm{r}^{\circ} \mathrm{a}-$ $30 r^{\circ}$ a ) Cette questıon est éditee dans Stefan Kırsc hner, Nicolaus Oresmes Kommentar zur Physık des Arıstoteles: Kommentar mit Editıon der Quaestıonen zu Buch 3 und 4 der Aristotelıschen Physık sowie von vier Quaestıonen zu Buch 5 (Stuttgart : Franz Steıner Verlag, 1997), "Sudhoffs Archıv Berhefte " 39, 197-206 Elle a été analysée par Stefano Carotı, La perceptıon du mouvement selon Nıcole ()resme, ın Comprendre et maîtrıser la nature au Moyen Âge Mélanges otferts à (juy Beaujouan (Genève : Droz, 1994), 83-99 Je ne présenteraı ı ı que quelques éléments de la seconde partıe de cette questıon non développes par Carotı, et je renvore pour le reste à son artıcle

14 - Il faut distınguer la lumière de la source lumıneuse appelée lux, de la lumıère créée dans le milieu appelée lumen. 
nécessite une comparaison, comparaison qui suppose l'intervention d'une virtus distinctiva (une puissance de distinguer) assimilée au sens commun d'Aristote. L'exemple qui est donné est celui de la connaissance de la grandeur d'une chose, qui résulte de la comparaison avec la grandeur unité, un pied par exemple. L'étude détaillée de cet exemple est faite dans la "Questio de apparentia rei ».

Le cadre perspectiviste étant rappelé, Nicole Oresme aborde le problème de la perception du mouvement en posant quatre suppositions (ou hypothèses), chacune suivie d'un ou plusieurs corollaires.

La première hypothèse expose comment est saisi le mouvement, celui-ci étant défini par le fait pour le mobile de se comporter autrement qu'avant ("aliter se habere quam prius») 15, et établit qu'il ne l'est ni par le sens extérieur (car tout ce que le sens perçoit, c'est la chose là où elle est), ni par le sens intérieur, mais moyennant un discours de l'intellect; ces trois modes de connaissance sont également décrits dans la "Questio de apparentia rei ». Grâce à sa virtus distinctiva, le sens intérieur peut percevoir, et ce sans erreur, que $A$ se comporte différemment de B ; si par ailleurs l'intellect sait que $A$ est au repos, il juge que $B$ est mû ; et si ce jugement est faux, par exemple si c'est A qui se meut, Oresme considère que l'erreur $\mathrm{n}^{\prime}$ est pas dans les sens mais dans le discours de l'intellect.

Inversement le repos est défini par "se comporter comme avant ". Dans le cas d'un mouvement correspondant à une différence de comportement trop faible pour être perçue, l'intellect peut juger qu'il y a repos. Paradoxalement, Oresme considère là encore que l'erreur est dans le discours de l'intellect.

La seconde hypothèse fait intervenir la théorie des species. On sait que pour expliquer la perception visuelle d'un objet distant, les perspectivistes admettaient qu'une suite d'images dudit objet, les species, était engendrée de proche en proche dans le milieu, depuis l'objet jusqu'à l'intérieur de l'œil ; ce processus était appelé la "multiplication des species". Il permettait de considérer que la perception se faisait par contact, celui de l'organe du sens et d'une

15 - Cette définıtıon est habıtuellement consıdérée comme la définıtıon burıdanıenne. Carotı montre que l'attrıbutıon, due à Annelıese Maıer, est erronée, et que cette définıtıon est reprise de Vitellıon. Voır Carotı, op cit in n. 13, 88-89 
species ${ }^{16}$. Remarquons que cette théorie permet aussi d'expliquer pourquoi le mouvement d'un objet A qui vient droit vers l'œil est perçu : même si la species qui est dans l'œil reste au même endroit, elle s'agrandit, et son comportement par rapport à l'œil est modifié.

Les deux dernières hypothèses rappellent que ce n'est que dans un temps, et non dans un instant, qu'on peut percevoir un mouvement, c'est-à-dire que quelque chose se comporte autrement qu'avant, et enfin que ce temps doit être perceptible.

Toutes ces hypothèses se situent dans le cadre perspectiviste, et sont conformes à ce qu'écrit Vitellion ${ }^{17}$. Elles permettent à Oresme d'imaginer deux expériences de pensée dans lesquelles un changement discontinu serait perçu comme un mouvement continu :

1/ quand un temps est divisé en un grand nombre d'intervalles imperceptibles et que le mobile se meut et repose alternativement dans ces intervalles;

2/ quand des instants sont séparés par des temps imperceptibles et qu'à chacun de ces instants le mobile saute instantanément d'un lieu à un autre immédiatement proche.

Dans la "Questio de apparentia rei », ces mêmes expériences sont données, ainsi que d'autres, plus élaborées. Nous reviendrons plus loin sur leur portée et leur signification.

Nicole Oresme introduit alors une distinction sur le sens de « je vois que $A$ est mû »: soit, dit-il, je sais par expérience et de façon certaine que $A$ est mû, de sorte que je le juge évident, soit je juge à partir de l'expérience visuelle que A est mû, et il me semble qu'il en est bien ainsi quoique ce ne soit pas évident. Cette même distinction est posée à propos de la grandeur dans la "Questio de apparentia rei ». Oresme semble alors quitter le domaine de la

16- Au XIV sıècle, la référence pour la théorıe des species est le tratté sur la multıplicatıon des espèc es de Roger Bacon, édité dans David L ınberg (ed.), Roger Bacon's phılosophy of nature A critic al edition, with English translation, introduction and notes, of the De multiplic atione spec ierum and De speculis comburentıbus, ed, transl and notes by David L ınberg (South Bend, Indıana 'St Augustıne's Press, 1998) Sur les discussions à propos des species, voir Leen Spruit, Species intelligibilis . From perception to knowledge Classical roots and medieval discussions (Leiden - New York - Cologne Brıll, 1994) Voır aussı à propos de l'opinıon d'Oresme, Claude Ciagnon, Le statut ontologique des species in medio chez Nicole Oresme, Arc hives d'historre doctrinale et litteraure du Moyen Âge, 60 (1993), 195-205

17 - Les références à Vitellıon dans les questıons sur la Physıque d'(Oresme, lıvres III et IV, sont donnees dans Kirschner, op cit in n 13, Index, 484 
connaissance sensible pour celui de la connaissance intellective, et particulièrement dans les deux conclusions qui suivent ${ }^{18}$.

La première énonce que nous n'avons pas une connaissance certaine et évidente résultant de l'expérience du mouvement d'une chose. Car être mû c'est se comporter maintenant différemment d'avant et après différemment de maintenant ( $A$ movetur » equivalet huic copulative " $A$ immediate ante movebatur et $A$ immediate post hoc movebitur ») or si nous pouvons voir que quelque chose est autrement qu'avant, nous ne pouvons pas avoir d'expérience immédiate du comportement futur de $\mathrm{A}$.

Aux objections qui disent que l'expérience nous montre que Socrate court, le grave tombe, le feu chauffe et le ciel se meut, Oresme répond qu'en réalité nous voyons Socrate courir dans un temps passé très proche de maintenant et que comme on sait qu'il ne peut s'arrêter brutalement, nous concluons qu'il se meut; de même nous savons qu'habituellement le feu chauffe, et nous savons non seulement par expérience mais aussi par raison que le ciel est mû. Finalement, ce sont la raison et une conjecture vraisemblable, et non l'intuition, qui nous permettent de dire que quelque chose est mû.

L'autre conclusion énonce que par l'expérience nous jugeons que $\mathrm{A}$ est mû, mais la connaissance que nous avons de ce mouvement n'est pas évidente : nous voyons A maintenant en un autre lieu qu'avant et nous jugeons qu'il en est ainsi, mais il n'est pas évident que cela soit vrai. Les expériences de pensée données plus haut sont aussi invoquées à l'appui de la conclusion.

À la suite de ces deux conclusions, Oresme observe qu'un mouvement ne peut être connu intuitivement parce qu'une telle connaissance ne concerne que le présent (seule la différence de choses simultanées est observable), mais seulement abstractivement ${ }^{19}$. Le renvoi à la distinction connaissance intuitive/connaissance abstractive est explicite.

Cette distinction est reprise par la plupart des protagonistes du débat sur la théorie de la connaissance au XIV ${ }^{\mathrm{e}}$ siècle, même s'ils ne

18 - Pour la clarté de l'exposé, je ne parle que de ces deux conclusions qui sont en fait les deuxı̀̀me et troısıème de la seconde partıe de la questıon Voır Kırschner, op cıt in n. 13, $201-203$.

19 - "Ex hoc sequitur corrolarie quod motus non cognosc itur cognitıone intuitıva, qua talıs cognitıo est solum de presentı, sed abstractıva "(Kırschner, op (it in n 13, 204 ) 
donnent pas exactement le même sens aux deux types de connaissance.

$C^{\prime}$ est à Duns Scot qu'on en attribue la première théorisation ${ }^{20}$. En résumant grossièrement : Scot considère que la connaissance obtenue à partir de la vision sensible, qu'il appelle connaissance abstractive, est une connaissance incomplète. Elle ne peut pas seule donner une certitude du fait qu'elle est médiatisée par la multiplication des species or, dit-il, les species peuvent représenter des choses existantes aussi bien que non-existantes. Pour le comprendre, il faut se souvenir qu'au cours du processus, une intervention surnaturelle est toujours possible, intervention qui pourrait par exemple conserver la species qui est dans I'œil et annihiler l'objet vu, voire éventuellement certaines des autres species.

Pour garantir la possibilité d'une connaissance certaine, Duns Scot affirme que si l'objet existe et est présent, l'observateur en a une connaissance immédiate et directe. Insistons sur le fait que l'objet n'est pas seulement connu en tant qu'objet singulier, il l'est dans son essence, comme concept : quand j'ai Gérard Simon devant moi, je sais immédiatement et de façon évidente qu'un homme existe. C'est cette connaissance immédiate qui est appelée intuitive.

Dans une telle théorie, la certitude de l'existence d'un objet est possible en ce monde, mais un processus du type de celui qui est décrit par les perspectivistes est incapable de l'assurer.

On peut alors se demander jusqu'à quel point Oresme s'inspire de Scot quand il oppose comme il vient de le faire connaissance intuitive et connaissance abstractive. Un élément de réponse est peut-être fourni par la fin du raisonnement précédent. Oresme observe que pour que le mouvement soit vraiment perçu, il faudrait que le sens, intérieur bien sûr, soit pourvu d'une puissance qui le rendrait capable de comparer présent et passé. Et ceci est finalement cohérent avec ce que dit Vitellion. II n'est plus question de discours de l'intellect comme dans les hypothèses préliminaires.

II semble qu'on puisse alors conclure qu'Oresme n'entre pas vraiment dans les discussions sur la connaissance intellective, et qu'il reste dans le champ d'une connaissance sensible étendue au cas

20) - Après Duns Scot, les conditıons vorre l'existence d'une telle connaissance intuitıve sont tres disc utees Voir Tachau, op cit in n 3, part II, 85-153 
imaginaire où les sens auraient une puissance de comparaison excédant celle de la vision immédiate.

Pour Oresme, connaissance intuitive semble à peu près synonyme de connaissance certaine et connaissance abstractive de connaissance sensible et incertaine. Et ce qu'il établit au terme de la question est que nous ne pouvons jamais avoir de connaissance certaine de l'existence d'un mouvement.

\section{La question "De apparentia rei »}

Comme il a été dit plus haut, le problème posé est ici celui de la possibilité de voir une chose aussi grande qu'elle est ${ }^{21}$.

Le thème est classique. II est traité ou au moins abordé dans les traités de perspective comme dans les textes traitant de la connaissance en général.

La question commence par des suppositions semblables à celles que nous avons vues au début de la question précédente :

- connaître une mesure, c'est connaître un rapport à une grandeur de référence ;

- on peut juger d'une grandeur de trois façons : soit par la vue seulement, soit au moyen d'une comparaison à l'aide d'une virtus distinctiva, soit par un discours de l'intellect (discursus mentis).

Oresme l'explique ici de la façon suivante : les sens dits inexpérimentés jugent des grandeurs des choses selon le rapport des angles sous lesquels on les voit ; c'est la première façon. Les sens expérimentés jugent de la grandeur des choses en connaissant en plus leur distance à l'observateur; c'est la seconde façon. Enfin l'intellect juge, par exemple de la grandeur du Soleil, au moyen d'un savoirfaire fondé sur les expériences des sens et certains principes vrais par soi ; c'est la troisième façon.

De façon assez semblable à ce que nous avons vu dans la question précédente avec le mouvement, la première série de conclusions va

21 - "Utrum alıqua res videatur tanta quanta est " J'a établı le texte à partır des deux manuscrits Venıse, bıblıotec a Marciana VIII 19, $234 \mathrm{r}^{\circ}-242 v^{\circ}$, et Séville, biblıoteca capitular Colombına $7713,138 \mathrm{r}^{\circ}-140 \mathrm{v}^{\circ}$ Maıs (es deux manusc rits sont des copıes appartenant à l'évidence à une même traditıon, sı bıen qu'un travaıl d'éditıon néc essıterait la prise en compte du texte abrégé conservé clans le manusc rit Erfurt, bıblıothèque de l'université, collection amplonıenne (Q 231, $138 \mathrm{r}^{\circ}-140 \mathrm{v}^{\circ}$ 
permettre d'établir qu'aucun jugement, qu'il soit du premier, du second ou du troisième mode, ne permet de savoir exactement quelle est la grandeur d'une chose.

Il commence par l'établir pour un processus de type abstractif (premier et second modes) en le prouvant de la façon suivante : quand, par la vue assistée d'une virtus distinctiva, nous jugeons de la grandeur d'une chose, puis quand nous acquiesçons à ce jugement, nous ne pouvons avoir aucune certitude ; en effet, le jugement est établi à l'aide de l'angle sous lequel on voit l'objet et cet angle n'est pas exactement connu car, si une chose s'éloigne continûment de l'œil, la diminution de l'angle sous lequel on la voit ne sera perceptible qu'à partir d'un certain éloignement. Notons qu'Oresme considère ici que c'est la virtus distinctiva qui est trompée, et donc le sens interne, et non comme il le dit dans la question précédente le jugement de l'intellect.

La propriété qui a été établie pour les deux premiers modes reste vraie pour le troisième mode, c'est-à-dire par le jugement de l'intellect, car celui-ci s'appuie toujours sur les sens.

Tout ce que permettent les sens, et donc l'intellect, c'est d'obtenir une estimation approchée comme moins de quatre pieds, ou à peu près trois pieds, c'est-à-dire sans différence sensible avec une mesure de trois pieds.

Une objection est alors envisagée: même si une grandeur en excède une autre insensiblement, il est possible à l'intellect par un raisonnement $d$ 'avoir connaissance de cette inégalité. Ainsi si on suppose que $A$ est plus grand que $C$ par un excès sensible dont la moitié est insensible, $B$ étant plus grand que $C$ par cet excès moitié, comme $B$ paraît égal à $C$, I'observateur qui sait que $A$ est plus grand que $C$ saura que $A$ est plus grand que $B$; par le même raisonnement il saura que $C$ est plus petit que $B$.

Mais cette objection est aussitôt réfutée au motif qu'il faudrait admettre que toutes les opérations décrites se font exactement alors que dans la pratique il y a des inégalités si faibles qu'elles ne sont pas perceptibles.

La conclusion est donc bien qu'il y a des inégalités qu'on ne peut pas connaître, des rapports (i. e. des mesures) qu'on ne connaît pas exactement. Et s'il arrive qu'on sache qu'une chose est de plus (ou 
de moins) de trois pieds, on ne sait jamais exactement quand elle est de trois pieds.

En fait il y a même des raisons mathématiques pour lesquelles on ne peut, de quelque façon que ce soit, juger de la grandeur de certaines quantités. Et Oresme donne l'exemple d'une grandeur dont le rapport à la grandeur de référence est irrationnel et sans dénomination connaissable, et il fait ici une assimilation, problématique, entre rapport connaissable et rapport de dénomination connaissable. En fait la référence est à ce qu'il écrit dans son traité Des rapports de rapports : seuls ont des dénominations connaissables les rapports rationnels et les rapports irrationnels dont nous dirions aujourd'hui qu'ils s'expriment comme puissances rationnelles de rapports rationnels. Et Oresme affirme dans le traité qu'il existe des rapports irrationnels qui ne sont pas de ce dernier type et donc sont sans dénomination connaissable ${ }^{22}$. Comme il I'a fait à propos du mouvement, Oresme a réfuté théoriquement la possibilité d'une connaissance certaine de la grandeur de certaines choses.

\section{Les expériences imaginaires}

Suivent alors cinq conclusions paradoxales décrivant des expériences visuelles imaginaires, mais possibles par la puissance divine, qu'il est intéressant d'examiner en détail car elles fournissent de bonnes illustrations de la méthode oresmienne. Elles relèvent de ce qu'on appelle la physique selon l'imagination.

Elles s'appuient sur trois suppositions qu'on trouve également dans $d^{\prime}$ autres œuvres d'Oresme.

1/ Une même chose peut, du fait de dispositions diverses des sens, apparaître tantôt plus grande tantôt plus petite : ceci est illustré par le fait qu'une même chose apparaît plus petite à un adulte qu'à I'enfant qu'il était. Oresme l'explique - mais l'explication est assortie d'un " peut-être » (forte) - en faisant correspondre l'apparence plus grande ou plus petite à la petitesse ou la grandeur de l'organe.

2/ Un même temps apparaît plus long ou plus court suivant les mouvements de l'imagination. L'exemple, classique, est celui des

22 - Cf. Nicole Oresme, De proportıonıbus proportıonum and Ad pauc a respic ientes, ed, transl. and notes by Edward Grant (Madison-Milwaukee-London - The Univ of Wisconsin Press, 1966), 160-166 
dormeurs de l'île de Sardaigne qui n'ont pas perçu l'écoulement du temps durant leur sommeil.

3/ Le temps, comme toute quantité continue, doit, pour être perçu, avoir une certaine grandeur ; autrement dit, il existe des minima temporels perceptibles.

Les deux premières conclusions suivent la première supposition, les deux suivantes la seconde supposition, la dernière la troisième supposition.

\section{Première conclusion}

Il est possible qu'une chose apparaisse plus grande à Socrate qu'à Platon, mais que toutefois elle leur apparaisse aussi grande qu'elle est.

En effet il est possible que, du fait des dispositions de leurs yeux, Socrate voie systématiquement une chose plus grande que Platon ne la voit, en d'autres termes que, par une puissance commune, la chose telle que vue par Socrate soit jugée plus grande que la chose telle que vue par Platon. Mais une chose jugée comme étant de trois pieds par Socrate sera également jugée de trois pieds par Platon car une mesure est un rapport et ce rapport est le même dans les deux visions, celle de Platon et celle de Socrate.

\section{Seconde conclusion}

On suppose que tous les corps sensibles sont diminués dans le rapport 2, et que les corps sont disposés de telle sorte que, les corps restant constants, les apparences sont multipliées par deux, par exemple si leurs organes sont diminués dans le même rapport ; dans ces conditions, aucune diminution ne sera perçue et tous les corps apparaîtront comme ils apparaissaient avant.

En conséquence Dieu pourrait, par une diminution continue pendant un temps fini, disons une heure, annihiler le monde sans que soit perçu autre chose qu'une annihilation instantanée à l'instant final.

En effet, dans la première moitié du temps (ce qu'on appelle la première partie proportionnelle du temps), Dieu peut tout diviser par 2, y compris les organes des sens, puis recommencer dans la 
première moitié de la moitié restante (la seconde partie proportionnelle) et ainsi de suite. Conformément à ce qu'a établi la seconde conclusion, pendant chacune des parties proportionnelles de I'heure, aucune diminution ne sera perçue ; mais au dernier instant l'observateur percevra une annihilation, instantanée donc.

\section{Troisième conclusion}

Si tous les mouvements du monde (chute des graves, mouvement du ciel, etc.) avaient leurs vitesses doublées, et si simultanément les imaginations de tous les hommes étaient configurées de telle sorte que le temps leur apparaisse deux fois plus lent, aucun changement ne serait perçu.

Il en serait de même pour un ralentissement ou pour une modification irrégulière des mouvements et une modification concomitante des imaginations, ou pour un ralentissement continu du mouvement du ciel jusqu'au repos, celui-ci toutefois étant exclu.

\section{Quatrième conclusion}

Dieu peut faire qu'après ce jour suivent mille années qui paraissent ne durer qu'un jour. Et Dieu peut même faire que Socrate ne dure qu'un jour et qu'il lui semble que ce jour dure un temps infini. En effet, il suffit que Dieu modifie convenablement les dispositions de l'âme des observateurs. Par exemple, si Socrate ne dure qu'un jour, Dieu peut faire que chacune des différentes parties proportionnelles du jour (dont les durées sont successivement une demi-journée, un quart de journée, etc.) lui paraissent durer comme la première, c'est-à-dire une demi-journée ; la durée totale lui apparaîtra alors infinie.

\section{Cinquième conclusion}

Il peut apparaître à un observateur convenablement disposé qu'un corps soit dans un lieu pendant une heure, alors qu'en réalité il ne l'est que pendant 1000 parties de cette heure séparées par des temps imperceptibles.

L'exemple (classique) est celui de la torche allumée qu'on fait tourner et qui est perçue comme un cercle brillant continu. II est repris sous une autre forme : celle d'un point qui parcourt une ligne 
$B C$ en allant et revenant très rapidement, de sorte qu'il paraît occuper tout l'espace BC; il paraît alors être en un point quelconque $D$ de $B C$ pendant toute l'heure, alors qu'il ne l'est que pendant un temps qui, tout entier, ne serait qu'un 1/1 $000 \mathrm{~d}^{\prime}$ heure, ou pourrait même être insensible.

Comme Dieu pourrait donner l'existence à Socrate puis I'annihiler à chaque fois que le point passe en D, Socrate pourrait n'exister que pendant un temps total équivalent à $1 / 1000 \mathrm{~d}$ 'heure et, pour un observateur, il apparaîtrait exister continûment pendant une heure ou un jour, etc.

Oresme reprend alors l'expérience de pensée donnée dans la question précédente, conséquence également de la troisième supposition : il est possible qu'un observateur juge qu'une chose est mue continûment pendant une heure alors que pendant cette heure elle ne subit qu'une suite de changements instantanés et donc n'est pas mue.

Deux situations imaginaires conséquences elles aussi de la troisième supposition sont alors décrites.

\section{Première situation}

Supposons qu'une heure soit divisée en un grand nombre de parties trop petites pour être perceptibles, disons 1000 parties. On suppose également que Dieu fasse que Socrate soit dans un lieu $A$ dans toutes les parties d'ordre impair et ne le soit pas dans toutes les parties d'ordre pair, il paraîtra alors être continûment en A pendant toute l'heure. Inversement on suppose que Platon soit dans le même lieu A pendant les parties d'ordre pair de l'heure et ne le soit pas dans les parties d'ordre impair ; lui aussi paraîtra être continûment en A pendant I'heure. On en déduit que deux corps, Socrate et Platon, paraîtront être ensemble dans le même lieu A.

\section{Seconde situation}

Soit une heure divisée par imagination en 1000 parties imperceptibles par exemple. Si pendant les parties d'ordre pair de l'heure, une proposition est vraie, par exemple "un corps A est blanc ", et si pendant les parties d'ordre impair, la contradictoire est vraie, "le même corps A n'est pas blanc ", les deux paraîtront vraies ensemble pendant toute I'heure. 


\section{Interprétations}

Quel sens doit-on donner à cette série de conclusions ?

Comme on l'a vu, les situations envisagées sont imaginaires mais elles $\mathrm{n}^{\prime i m p l i q u e n t ~ p a s ~ a ~ p r i o r i ~ d e ~ c o n t r a d i c t i o n s ~ l o g i q u e s, ~ c o m m e ~}$ le ferait une situation où, par exemple, une chose serait et ne serait pas au même lieu au même instant, ou deux choses seraient simultanément dans le même lieu. Elles ne font intervenir que des créations et annihilations de substances, créations et annihilations qui sont toujours possibles par la puissance divine.

L'intervention de telles imaginations n'a rien d'original dans un contexte gnoséologique: dans le débat sur la différence entre connaissance intuitive et connaissance abstractive, Duns Scot soutient que l'objet de la première, la connaissance intuitive, devrait être présent et existant, et l'objet de l'autre, la connaissance abstractive, pas nécessairement; à quoi Pierre Auriol 23 réplique que Dieu pourrait supprimer une étoile et faire que l'intellect de l'observateur soit dans les mêmes dispositions qu'avant la suppression, si bien qu'il aurait de l'étoile la même connaissance intuitive que si elle était présente. L'exemple, quoi qu'on en pense aujourd'hui, était une de ces situations naturellement impossibles et possibles seulement par la puissance divine.

L'autre caractère remarquable est le recours aux mathématiques, et notamment aux mathématiques de I'infini, avec une utilisation presque systématique de la division en parties proportionnelles.

Cas imaginaires possibles par la puissance divine, et recours aux mathématiques (et aux subtilités logiques), sont deux des caractéristiques de cette physique imaginaire mise à l'honneur par les physiciens d'Oxford et adoptée dans les années 1340 par certains maîtres parisiens dont Oresme est généralement considéré comme la figure dominante.

Que peut-on attendre d'une telle physique? Les inférences étant correctes, puisque les prémisses, c'est-à-dire les cas imaginaires proposés, sont possibles, même si c'est au sens où elles ne sont pas contradictoires, les conclusions le sont également, même si elles paraissent paradoxales.

23 - Sur la gnoséologıe d'Aurıol et son oppositıon à Duns Scot, voır Tachau, op cit in n. 3 , 85-112 
Dans les textes gnoséologiques, des exemples d'illusions visuelles sont couramment évoqués, mais ils sont très répétitifs ; reviennent constamment le bateau dont les occupants voient la rive en mouvement, la torche qu'on fait tourner et un très petit nombre d'autres. Le recours d'Oresme aux cas imaginaires augmente considérablement le nombre des illusions envisageables.

Nos cinq conclusions sont articulées sur des cas de ce type et sont destinées à explorer la notion de perception de la grandeur, du temps et surtout du mouvement, thème de prédilection pour Oresme comme on l'a vu dans la première question décrite, mais aussi dans d'autres traités comme Le Traité du ciel et du monde. Notons bien que ce qu'Oresme explore, c'est la notion de perception du mouvement, pas le mouvement lui-même : dans la question III.1, il démontre simplement qu'on ne peut jamais avoir la certitude qu'un corps est en mouvement, et qu'une connaissance certaine en est impossible.

Rappelons enfin que caractère paradoxal des propriétés et exploration de notions physiques sous-jacentes sont deux traits caractéristiques de la littérature appelée sophismatique, qui s'est considérablement développée dans la première moitié du XIV" siècle.

\section{Conclusions}

À partir de ce survol que peut-on dire de la gnoséologie d'Oresme ?

Comme il a été dit plus haut, et bien qu'il ne lui ait consacré spécifiquement aucun ouvrage, le thème de la connaissance a une place importante dans son œuvre. La position qu'il soutient constamment est que la connaissance des choses procède toujours des sens : Ia seule connaissance est la connaissance sensible abstractive, et elle n'est jamais certaine. C'est ce qu'il montre dans nos deux questions à propos du mouvement et de la grandeur, dans le De visione stellarum, à propos de la position des astres, et plus nettement encore dans la question I.2 où il affirme que la seule connaissance qu'on ait de la substance est celle qui, par une déduction logique, résulte de la connaissance des accidents, connaissance elle aussi incertaine puisque provenant d'une perception sensible. Comme on l'a vu dans nos deux questions, les hypothèses et les arguments se retrouvent d'un texte à I'autre, avec des variantes peu nombreuses ; on en a signalé une sur l'origine de certaines erreurs visuelles. 
Tout ceci pourrait sembler légitimer la qualification de sceptique qu'on lui a attribuée ${ }^{24}$. Et pourtant peut-on vraiment considérer qu'est sceptique quelqu'un qui considère que la raison humaine appuyée sur la science la plus certaine, les mathématiques, a accès à une physique imaginaire possible par la seule puissance divine, physique imaginaire qui a des résonances dans les débats gnoséologiques de l'époque? L'exemple le plus net est celui de la controverse autour des thèses de Nicolas d'Autrécourt, condamnées en 1346, car les deux dernières situations imaginaires évoquées peuvent être mises en rapport avec certaines des thèses de ce dernier.

Dans sa théorie de la connaissance, celui-ci fait jouer un rôle particulier aux apparences. Comme Oresme, il n'envisage qu'un seul mode de connaissance, celui qui est fondé sur la perception sensible. Mais contrairement à ses prédécesseurs, il ne considère pas qu'une apparence soit dans l'âme, ou si elle est hors de l'âme, qu'elle ait un degré d'être diminué. Pour lui, les apparences sont tout à fait réelles, elles sont en quelque sorte externalisées, et ne sont pas le produit d'actes cognitifs. Avec elles commencent et finissent notre conscience et notre connaissance du monde, et à partir d'elles, il ne nous est possible de déduire l'existence d'autres choses réelles, substance ou autre, qu'en référant au premier principe ou principe de contradiction, c'est-à-dire en établissant que la non-existence de ces autres choses est contradictoire avec l'apparence perçue ${ }^{25}$.

Cette doctrine a des points communs avec celle d'Oresme mais aussi de grandes différences, et les conclusions tirées des dernières situations imaginaires envisagées dans la "Questio de apparentia rei " semblent bien viser Nicolas d'Autrécourt. Car si les apparences sont des réalités, ce sont deux réalités, I'apparence de Socrate et celle de Platon, qui seront réellement ensemble dans le même lieu,

24 - Voir Edward Grant, Scientific thought in fourteenth-century Paris - Jean Buridan and Nicole (Oresme, in Madeleıne Pelner Cosman and Bruce Chandler (ed ), Machault's world - Science and art in the fourteenth century (New York - New York Academy of sciences, 1978), 105-124 L'artic le est repris dans Edward Giant, Studies in medieval science and natural phılosophy (London - Varıorum Reprınts, 1981) Cirant oppose le "physicien "Jean Burıdan qui s'attac he à établır les fondements de la connaıssance de la vérité sur le monde physique au "sceptıque "Nicole ()resme Le thème plus général du sceptıcısme de la phılosophıe du XIV" sıèc le a été abordé par Konstanty Mıc halskı, La Phılosophıe du XIV" sıècle - Six études, réımpr (Frankfurt am Maın · Mınerva, 1969), et critıqué par Lambert Marıe De Rıjk, La Phılosophie au Moyen-Âge (L eiden Brill, 1985), 215-218. De Riık propose de remplac er "sceptic isme " par " critic isme "

25 - Voir à ce sujet Denery, op c it in n 3, 156-158 
et de même deux propositions contradictoires qui, ensemble, seront vraies. Ce dernier exemple n'est pas pris au hasard car il va directement contre le premier principe, ou principe de noncontradiction, sur lequel d'Autrécourt fonde toute sa gnoséologie.

Les cas envisagés ne seraient donc pas posés gratuitement - par " esbatement ", dirait Oresme - mais bien pour réfuter les thèses de Nicolas d'Autrécourt.

Finalement si on juge utile de caractériser par un mot la gnoséologie d'Oresme, il faut certes prendre en compte l'incertitude de la perception sensible, mais aussi la revendication de la possibilité d'élargissement du champ d'intervention de l'intellect et le recours privilégié au raisonnement mathématique, le raisonnement le plus certain. Doit-on alors parler de scepticisme ou de rationalisme? 